\title{
Characterization of the Millimeter-Wave Polarization of Centaurus A with QUaD
}

\section{Citation}

Zemcov, Micahel B., Peter Ade, James Bock, Melanie Bowden, Micahel L. Brown, Gary Cahill, Patricia G. Castro, and et al. 2010. Characterization of the millimeter-wave polarization of Centaurus A with QUaD. The Astrophysical Journal 710, no. 2: 1541-1550.

\section{Published Version}

doi:10.1088/0004-637X/710/2/1541

\section{Permanent link}

http://nrs.harvard.edu/urn-3:HUL.InstRepos:11129151

\section{Terms of Use}

This article was downloaded from Harvard University's DASH repository, and is made available under the terms and conditions applicable to Other Posted Material, as set forth at http:// nrs.harvard.edu/urn-3:HUL.InstRepos:dash.current.terms-of-use\#LAA

\section{Share Your Story}

The Harvard community has made this article openly available.

Please share how this access benefits you. Submit a story.

\section{Accessibility}




\title{
CHARACTERIZATION OF THE MILLIMETER-WAVE POLARIZATION OF CENTAURUS A WITH QUaD
}

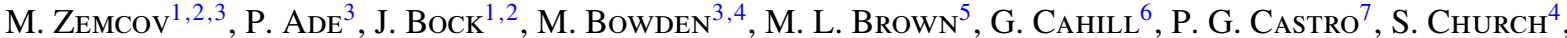 \\ T. Culverhouse ${ }^{8}$, R. B. Friedman ${ }^{8}$, K. Ganga ${ }^{9}$, W. K. Gear ${ }^{3}$, S. Gupta ${ }^{3}$, J. Hinderks ${ }^{4,11}$, J. Kovac ${ }^{2}$, A. E. Lange ${ }^{2}$, \\ E. Leitch ${ }^{1,2}$, S. J. Melhuish ${ }^{3,12}$, Y. Memari ${ }^{10}$, J. A. Murphy ${ }^{6}$, A. Orlando ${ }^{2,3}$, C. O’ Sullivan $^{6}$, L. Piccirillo $^{3,12}$, C. Pryke ${ }^{8}$, \\ N. Rajguru ${ }^{3,13}$, B. Rusholme ${ }^{4,14}$, R. Schwarz ${ }^{8}$, A. N. TAYlor ${ }^{10}$, K. L. Thompson ${ }^{4}$, A. H. Turner ${ }^{3}$, And E. Y. S. Wu ${ }^{4}$ \\ (QUAD COLLABORATION) \\ ${ }^{1}$ Jet Propulsion Laboratory, 4800 Oak Grove Dr., Pasadena, CA 91109, USA \\ ${ }^{2}$ California Institute of Technology, Pasadena, CA 91125, USA \\ ${ }^{3}$ School of Physics and Astronomy, Cardiff University, Queen's Buildings, The Parade, Cardiff CF24 3AA, UK \\ ${ }^{4}$ Kavli Institute for Particle Astrophysics and Cosmology and Department of Physics, Stanford University, 382 Via Pueblo Mall, Stanford, CA 94305, USA \\ ${ }^{5}$ Kavli Institute for Cosmology and Cavendish Astrophysics, University of Cambridge, Madingley Road, Cambridge CB3 OHA, UK \\ ${ }^{6}$ Department of Experimental Physics, National University of Ireland Maynooth, Maynooth, Co. Kildare, Ireland \\ ${ }^{7}$ CENTRA, Departamento de Física, Edifício Ciência, Piso 4, Instituto Superior Técnico - IST, Universidade Técnica de Lisboa, Av. Rovisco Pais 1, 1049-001 \\ Lisboa, Portugal \\ ${ }^{8}$ Kavli Institute for Cosmological Physics, Department of Astronomy \& Astrophysics, Enrico Fermi Institute, University of Chicago, \\ 5640 South Ellis Avenue, Chicago, IL 60637, USA \\ ${ }^{9}$ APC/Université Paris 7-Denis Diderot/CNRS, Bâtiment Condorcet, 10, rue Alice Domon et Léonie Duquet, 75205 Paris Cedex 13, France \\ ${ }^{10}$ Institute for Astronomy, University of Edinburgh, Royal Observatory, Blackford Hill, Edinburgh EH9 3HJ, UK \\ Received 2009 August 17; accepted 2009 December 30; published 2010 February 1
}

\begin{abstract}
Centaurus (Cen) A represents one of the best candidates for an isolated, compact, highly polarized source that is bright at typical cosmic microwave background (CMB) experiment frequencies. We present measurements of the $4^{\circ} \times 2^{\circ}$ region centered on Cen $\mathrm{A}$ with $\mathrm{QUaD}$, a CMB polarimeter whose absolute polarization angle is known to an accuracy of 0.5 . Simulations are performed to assess the effect of misestimation of the instrumental parameters on the final measurement and systematic errors due to the field's background structure and temporal variability from Cen A's nuclear region are determined. The total $(Q, U)$ of the inner lobe region is $(1.00 \pm 0.07$ (stat.) \pm 0.04 (sys.), $-1.72 \pm 0.06 \pm 0.05) \mathrm{Jy}$ at $100 \mathrm{GHz}$ and $(0.80 \pm 0.06 \pm 0.06,-1.40 \pm 0.07 \pm$ $0.08) \mathrm{Jy}$ at $150 \mathrm{GHz}$, leading to polarization angles and total errors of $-30.0 \pm 1.1$ and $-29.1 \pm 1.7$. These measurements will allow the use of Cen A as a polarized calibration source for future millimeter experiments.
\end{abstract}

Key words: cosmic background radiation - galaxies: individual (Centaurus A) - instrumentation: polarimeters radio continuum: general

Online-only material: color figures

\section{INTRODUCTION}

Cosmic microwave background (CMB) polarimeters require accurate calibration of both their temperature and polarization properties. Unfortunately, astronomical sources suitable for polarization calibration in the wavelength range at which CMB experiments typically operate are rare. With a number of exquisitely sensitive instruments in the field or near to deployment, the need for astrophysical polarization calibration sources useful for instruments with beam sizes of several to tens of arcmin is acute.

An ideal polarized calibration source would be compact, bright in both temperature and polarization, static over time, and isolated from galactic diffuse emission. Unfortunately, the typical frequencies at which CMB experiments are designed to measure are minima in the emission spectra for other astrophysical sources of radiation (synchrotron, free-free, and thermal dust emission), so such sources have not been identified

\footnotetext{
${ }^{11}$ Current address: NASA Goddard Space Flight Center, 8800 Greenbelt Road, Greenbelt, MD 20771, USA

12 Current address: School of Physics and Astronomy, University of Manchester, Manchester M13 9PL, UK

${ }^{13}$ Current address: Department of Physics and Astronomy, University College London, Gower Street, London WC1E 6BT, UK

${ }^{14}$ Current address: Infrared Processing and Analysis Center, California Institute of Technology, Pasadena, CA 91125, USA
}

in large numbers or studied in great detail at the frequencies of interest. A noteworthy candidate calibration source which has been observed with CMB polarimeters in the past is the supernova remnant Taurus A (Leitch et al. 2002; Barkats et al. 2005), though as an object in the northern hemisphere it is not available to telescopes at all sites.

One of the best candidates for a CMB polarization calibration source in the southern hemisphere is the radio bright galaxy Centaurus A (hereafter Cen A; Israel 1998 provides an excellent review of its multi-wavelength properties). The optical counterpart of Cen A is the massive elliptical galaxy NGC 5128 at a distance of 3.4 Mpc. At radio frequencies, Cen A presents rich structure over many decades in angular size driven by its nuclear source. Collimated radio jets are emitted from the compact nucleus and become sub-sonic a few parsec $\left(\sim 00^{\prime \prime} 1\right)$ from the central source. At $5 \mathrm{kpc}\left(\sim 4^{\prime}\right)$ from the nucleus, the jets expand into plumes which spread up to $250 \mathrm{kpc}\left(\sim 3^{\circ}\right)$ into the inter cluster medium (ICM). The interface between the bright sub-sonic jets and the smooth, low surface brightness plumes is abrupt, allowing high signal-to-noise measurements of the jets themselves. The $\sim 10^{\prime}$ jets, known as Cen A's inner lobes in the literature, have a spectral index of -0.7 from $>500 \mathrm{MHz}$ to $\sim 100 \mathrm{GHz}$ and are both polarized and bright at $v \gtrsim 100 \mathrm{GHz}$. Moreover, Cen A's inner lobes are unique as their properties do not vary on human timescales, which makes them perfect 
candidates for polarization calibration measurements. Cen A also lies well above the galactic plane, is well matched to the size of typical CMB polarimeter beam sizes, and is well studied at many other wavelengths, particularly in the radio. In this paper, we present the results of observations of Cen A with QUaD, a CMB polarimeter capable of simultaneously measuring Stokes' $I, Q$, and $U$ parameters with bands at 100 and $150 \mathrm{GHz}$ and angular resolution $5^{\prime} .0$ and 3.5, respectively.

\section{INSTRUMENT SUMMARY AND OBSERVATIONS}

A full description of the QUaD instrument can be found in Hinderks et al. (2009, hereafter H09). QUaD was a $2.6 \mathrm{~m}$ Cassegrain telescope using the pre-existing DASI mount at the US south pole station (Leitch et al. 2002). QUaD operated from 2005 February to 2007 November, taking astronomical data during the austral autumn through winter into spring; maintenance and calibrations were performed during the austral summer. The receiver comprised 31 pairs of polarization sensitive bolometers (PSBs), 12 pairs at $100 \mathrm{GHz}$ and 19 pairs at $150 \mathrm{GHz}$. At either frequency these were split into two polarization groups which measure either $Q$ or $U$; each bolometer pair is also sensitive to Stokes' $I$.

The data presented here were obtained on 2006 June 7 and 2007 October 2, 3, and 4. The observations were performed in a similar manner to a standard QUaD observation block as described in Pryke et al. (2009, hereafter P09), although the lead-trail scan strategy used in other QUaD observations is not employed. Each scan group begins with an elevation nod during which the telescope is moved up and down by $1^{\circ}$ in elevation to inject a signal of common amplitude into each bolometer. The telescope is then scanned back and forth at constant elevation over a throw of 3.2 in sidereal tracking corrected azimuth; four such scans constitute a single scan block. For these observations, the scan rate is 0.066 per second in azimuth, or 0.05 per second on the sky at Cen A's elevation. After each constant elevation scan block, the elevation is stepped by 1.2 arcmin and the next block is begun. In all, 101 scan blocks were performed, equating to a rastered data set mapping $\sim 4^{\circ} \times 2^{\circ}$ on the sky to a uniform noise level. These 101 scans required $15.6 \mathrm{hr}$; adding the standard QUaD calibration set before and after the scan block (see H09 for a description of such a set) leads to a total observation time of $17.6 \mathrm{hr}$ per day.

The DASI mount supports rotation of the entire telescope about its boresight; on a given day this rotation was fixed for the Cen A observations. However, the rotation was varied over different days, which has the benefit of changing the polarization characteristics of the instrument in a known manner. The angles chosen were $\left\{0^{\circ},-30^{\circ},+30^{\circ}, 0^{\circ}\right\}$ for 2006 June 7 and 2007 October 2, 3, and 4, respectively.

\section{FROM TIME STREAMS TO $T$ MAPS}

Unlike standard CMB observations, the QUaD polarization maps of Cen A require systematic effect corrections derived from the temperature maps (Section 4), so it is necessary to utilize a two-step process where the total intensity maps and then the polarization maps are constructed. The Cen A temperature maps themselves can be constructed directly from the data time series without any such corrections.

In order to produce maps, the relationship between the measured time series $d(t)$ and the sky temperatures $T, Q, U$ must be known. Neglecting noise contributions and the bolometer transfer function, it can be written as

$$
\begin{aligned}
d(t)= & g(t) \int d \mathbf{r} \mathcal{B}\left(\mathbf{r}-\mathbf{r}_{b}\right) \\
& \times\left[T(\mathbf{r})+\frac{1-\epsilon}{1+\epsilon}\{Q(\mathbf{r}) \cos 2 \psi+U(\mathbf{r}) \sin 2 \psi\}\right],
\end{aligned}
$$

where $\mathbf{r}$ is the direction of observation and $\psi$ is the detector's polarization angle (the time dependence of these two quantities is suppressed for clarity). The time-dependent gain $g$, cross polar leakage $\epsilon$, and polarized beam shape $\mathcal{B}$ are all quantities that must be determined via calibration measurements, as must be the zero point of $\psi$.

Initial low-level processing of the time series is performed using the same algorithm as presented in P09. The bolometer time constants and electronic filters are first deconvolved to reverse their effect on the time series. These time series are then despiked by removing those individual scans which exhibit transient impulse events from the analysis. The elevation nods are used to determine $g(t)$ for each scan block by using the changing atmospheric loading to compute the relative responsivity of each bolometer in a frequency group. Each detector in a frequency group is scaled to the group's mean, yielding relatively calibrated time series for the entire array.

Three more pieces of information are required to create maps from the rectified and responsivity calibrated bolometer time ordered data (TOD): the telescope pointing, the PSB angles, and the PSB efficiencies. Knowing the pointing of the telescope requires knowledge of both the sky offset of each of the detectors and the absolute pointing of the boresight, the derivation of these parameters is described in detail in H09. The detector offset angles used in the map making process are derived from monthly measurements and have an estimated uncertainty of 0.15 ; since the signal-to-noise ratio on Cen $\mathrm{A}$ is large the absolute per day pointing solution can be directly determined from I maps.

The PSB polarization angles and efficiencies were determined using a chopped thermal source placed behind a polarizing grid on a mast near the telescope; H09 reviews the measurement and preliminary analysis of these data. The cross polar leakage is defined as the ratio of response to anti-aligned and co-aligned incident polarized light for a given PSB. The measured values of $\epsilon$ have a mean of 0.08 with an rms scatter of 0.015 .

Knowledge of the error on the absolute polarization angle has improved over that presented in H09 thanks to both an improvement in the analysis of the calibration data and a new method of measuring this angle. The new method of angle measurement relies on the fact that the polarized response of the detectors is mechanically constrained to lie along lines of symmetry of the detector rows, any misalignment is estimated to be random and $<0.5$ per detector. Measurement of the polarization angle is performed by scanning rows of detectors across bright sources at telescope rotation angles where the polarized response of the detectors matches the orientation of the rows. As there are several such symmetries, a number of independent measurements can be made. The final error ascribed to this angle measurement method is 0.15 .

The improved analysis of the calibration source data presented in H09 is based on the observation that the orientation of the polarized source was not well constrained in the azimuthal plane; azimuthal misalignments at the source box can result in spurious polarization measured at the telescope. We therefore discard all measurements performed with the calibration source 

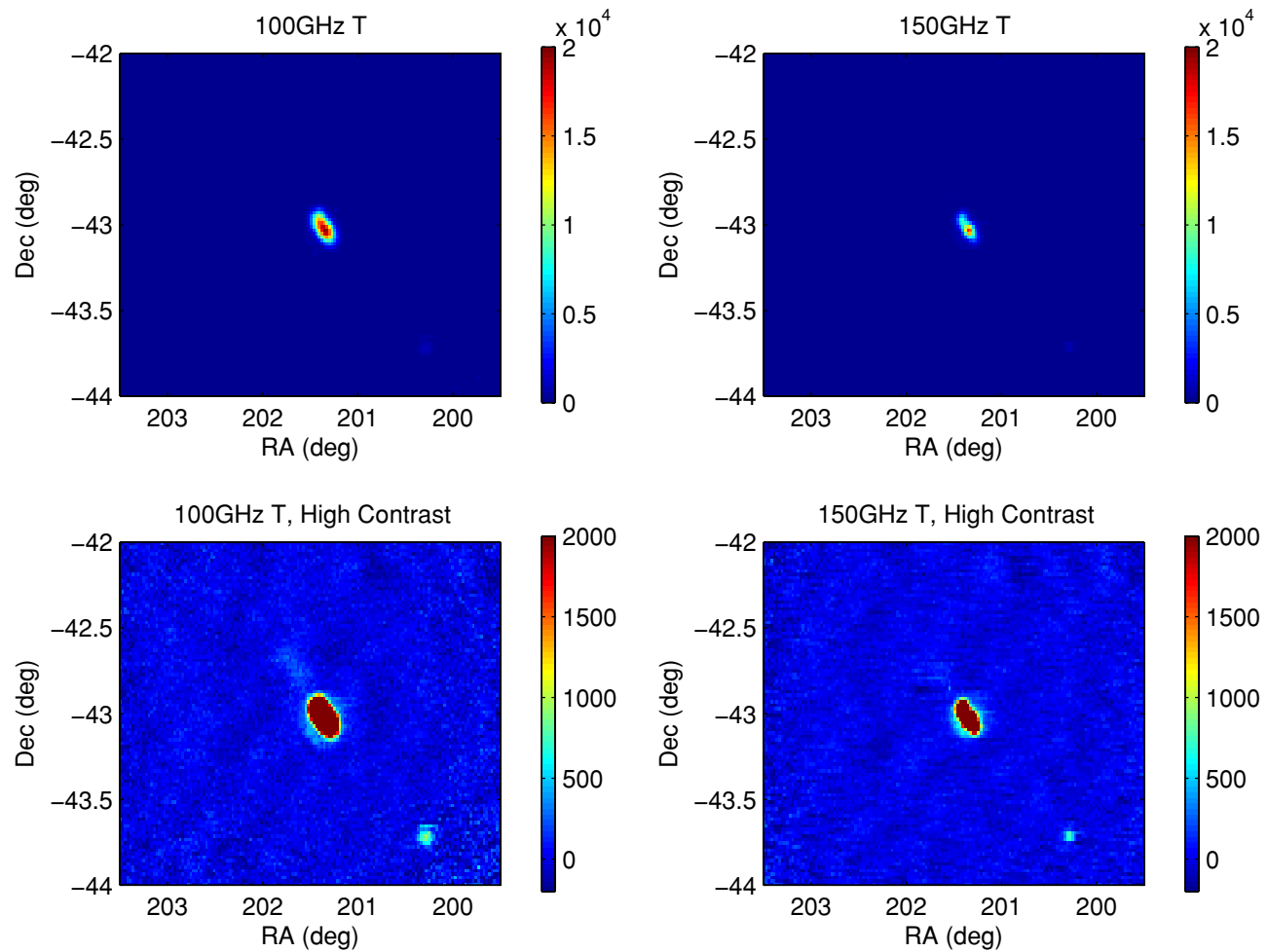

Figure 1. Temperature maps of Centaurus A. Temperature scale is $\mu \mathrm{K}_{\mathrm{CMB}}$. Top: $100 \mathrm{GHz}$ (left) and $150 \mathrm{GHz}$ (right) maps of the Cen A region at low contrast. The R.A. and decl. range has been chosen to facilitate comparison with Figure 3 of Israel (1998) and Figure 1 of Israel et al. (2008); none of the large-scale structure visible in those maps at lower frequencies is present in the $\mathrm{QUaD}$ data. The structure of the inner lobe region comprising two synchrotron emitting convexities in the ICM is unresolved at $100 \mathrm{GHz}$ but resolved at $150 \mathrm{GHz}$. Bottom: $100 \mathrm{GHz}$ (left) and $150 \mathrm{GHz}$ (right) maps of Cen A at high contrast. Low surface brightness emission is visible to the northeast of the inner lobe region, as is NCG 5090, an unrelated elliptical galaxy in the Cen A field near $\alpha_{\mathrm{J} 2000}=200^{\circ} .266, \delta_{\mathrm{J} 2000}=-43^{\circ} .741$.

(A color version of this figure is available in the online journal.)

grid in the horizontal orientation and use only the vertical grid measurements; these are immune to such alignment errors and have a well measured orientation with respect to gravity. The updated analysis which uses only the grid-vertical measurements has a scatter of 0.2 over the measurements. The absolute polarization angle of the telescope derived from these measurements is in good agreement with the angle derived from the row scans. We therefore use this revised absolute telescope angle and conservatively ascribe an error of \pm 0.5 to its measurement.

To construct maps further processing of the TOD is required. The PSB pair time streams are first summed to create total intensity TOD corresponding to the Stokes' parameter $I$ and differenced to make instrument frame Stokes' $Q$ or $U$; these must be rotated to an absolute reference frame for polarization using the known polarization angle of the telescope (as discussed in Section 4). Drifts in the atmospheric emission and instrument create $1 / f$ noise in the TOD much larger than photon noise on long timescales; to remove these correlated signals a fifth-order polynomial is fit to each $48 \mathrm{~s}$ half-scan and subtracted from the sum and difference TOD. This is a higher order than that chosen for the CMB data since we are not attempting to measure large spatial modes in the Cen A maps. As the signal-to-noise ratio on the source is large in a single scan, it is necessary to mask source flux to prevent biasing the polynomial fits. The mask used here comprises a circular region with $r=0.2$ centered on Cen A; this size was chosen to preserve structure in the inner lobe region while maximally removing drifts in the TOD. The same mask is used for the sum and difference TOD. When filtering the sum data to produce $I$ maps, the fit mask is also augmented by two circular regions centered at R.A. $=\left\{13.450,13^{\mathrm{h}} .353\right\}$, decl. $=\{-42.80,-43.70\}$; these mask Cen A's moderately bright northern spur region and the elliptical galaxy NGC 5090 from the polynomial filter.

Maps are made by binning the TOD into a grid of pixels weighting by the inverse variance of each half-scan computed after the polynomial filter has been applied. The map pixelization is in R.A. and decl. using square pixels 0.02 on a side. The per day signal-to-noise ratio in the I maps is large, so by comparing individual day maps it is possible to observe the telescope pointing solution vary by up to 30 arcsec day to day. To correct for this variation, the per day $I$ maps are made and that day's astrometric offsets are found by minimizing the sum of the squared pixel differences in the region of Cen A between that day's map and the map derived from the first day's observation with its astrometric calibration manually set to Cen A's known position. Once determined, these offsets are then always applied to both sum and difference TOD during the map making process.

To calibrate to $\mathrm{CMB} \mathrm{mK}$ we utilize the $\mathrm{CMB}$ power spectrum cross calibration procedure presented in Brown et al. (2009); the resulting temperature maps are shown in Figure 1. To calibrate between $I$ maps in $\mathrm{Jy} \mathrm{sr}^{-1}$ and $T$ maps in thermodynamic temperature the relation

$$
d I=(d B / d T)_{2.73 \mathrm{~K}} d T
$$

is used. These values correspond to $\{29.2,58.5\}$ Jy map pixel $^{-1} \mathrm{~K}^{-1}$ at $\{100,150\} \mathrm{GHz}$. 

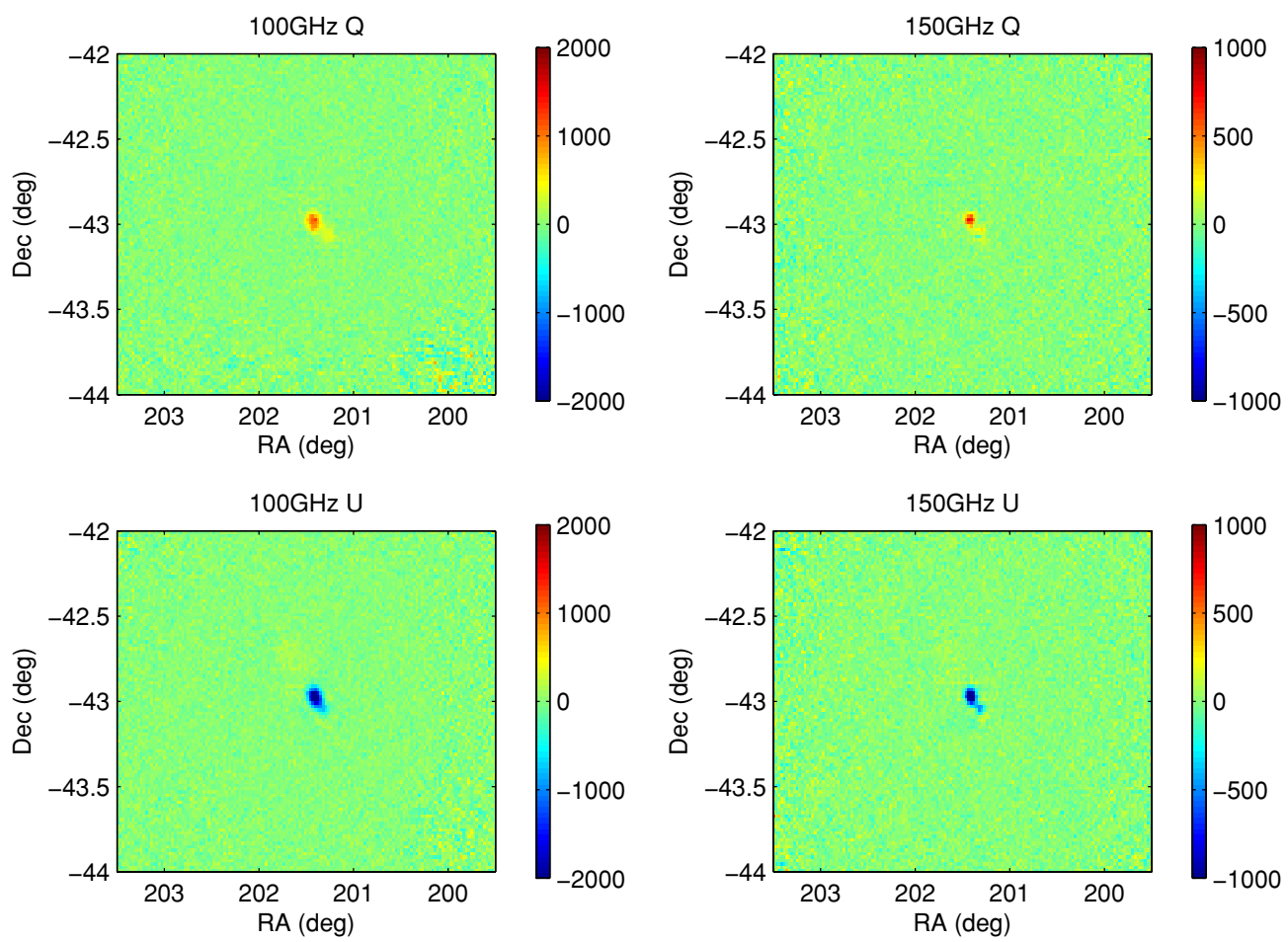

Figure 2. Polarization leakage corrected maps of Centaurus A. Temperature scale is $\mu \mathrm{K}_{\mathrm{CMB}}$. Top: $100 \mathrm{GHz}$ (left) and $150 \mathrm{GHz}$ (right) maps of the Cen A region for Stokes' $Q$. Bottom: $100 \mathrm{GHz}$ (left) and $150 \mathrm{GHz}$ (right) maps of the Cen A region for Stokes' $U$. The polarization of the northern inner lobe is about $20 \%$ of $I$ at either frequency. The polarization fraction in the southern lobe is much smaller.

(A color version of this figure is available in the online journal.)

\section{POLARIZED INSTRUMENTAL EFFECT CORRECTION}

There are a wide range of systematic effects which can mix $I$ to polarization and $Q$ to/from $U$ in polarimeters. The $\mathrm{QUaD}$ simulator, discussed in detail in P09, is an extremely detailed model of the instrument and various sources of noise whose primary purpose is to model time streams for use in MASTERstyle CMB power spectra analysis (Hivon et al. 2002). As the simulator is built to model the instrument accurately it can be used to measure and correct for instrumental systematic effects that are known to exist in QUaD data, whatever the astronomical source under study. In order to produce clean polarization maps, it is necessary to simulate and correct for these polarization mixing effects.

\subsection{Polarization Leakage Correction}

Although the detector offset angles show no evidence for variation over time or between bolometer pairs sharing a feed horn, they do exhibit repeatable offsets between the two halves of each detector pair with an rms magnitude of 0.1 over the array. When the sum and difference maps are constructed, the mean detector offset of a pair is used; unfortunately, the difference between this mean and the actual pointing of a detector can mix $I \rightarrow Q, U$. This polarization leakage can be quantified using simulations of the observations.

To measure polarization leakage, an input map is generated from the co-added $I$ maps at each frequency shown in Figure 1. Hereafter, we denote the "polynomial filter mask region" to be that area in the map corresponding to the regions masked in the time stream polynomial fit discussed in Section 3, i.e., the $r<0.2$ area centered on the source. Regions outside of the polynomial filter mask region are set to zero, such that only the central area contains non-zero flux. Each detector's pointing time stream is constructed using the individual measured offsets, which vary between detectors in a pair. These time series are used to sample a new, simulated time stream from the measured $I$ maps. As no noise is added to the TOD and the polarization maps are set to zero, this procedure generates a simulation of the pure I component of the Cen A observation for each detector. The PSB pairs are then differenced in the standard way; if the pointing between pairs was perfect, this procedure would cancel the structure appearing in the constructed $I$ maps perfectly. However, as the pair pointing is not identical, this procedure produces residuals reflecting the leakage of $I$ to polarization in the actual observation. These leakage time streams are subtracted from their corresponding pairs in the data TOD, which corrects for the leakage effects at the time sample level.

These polarization leakage corrected time streams are mapped onto the same R.A. and decl. grid as the $I$ data. For difference time stream data, the product of the data and the sine and cosine of the detector angles as projected on the sky must be accumulated for each pixel to weight the different detector pairs according to their polarization sensitivities; this procedure rotates instrument frame $Q$ and $U$ to absolute $Q$ and $U$. The set of $2 \times 2$ matrices comprising products of these sines, cosines, and difference time streams must be inverted for each pixel; these are then accumulated into the grid to produce absolutely referenced $Q$ and $U$ maps. In this work, we follow the IAU polarization convention with positive $Q$ running north-south and positive $U$ running northeast-southwest (Hamaker \& Bregman 1996). Figure 2 shows the CMB temperature calibrated $Q$ and $U$ maps at 100 and $150 \mathrm{GHz}$. These, combined with the $I$ maps, are the fundamental output of the analysis pipeline.

These maps can be used to characterize the polarization of Cen A per QUaD beam or per map pixel as desired (see Section 5). 

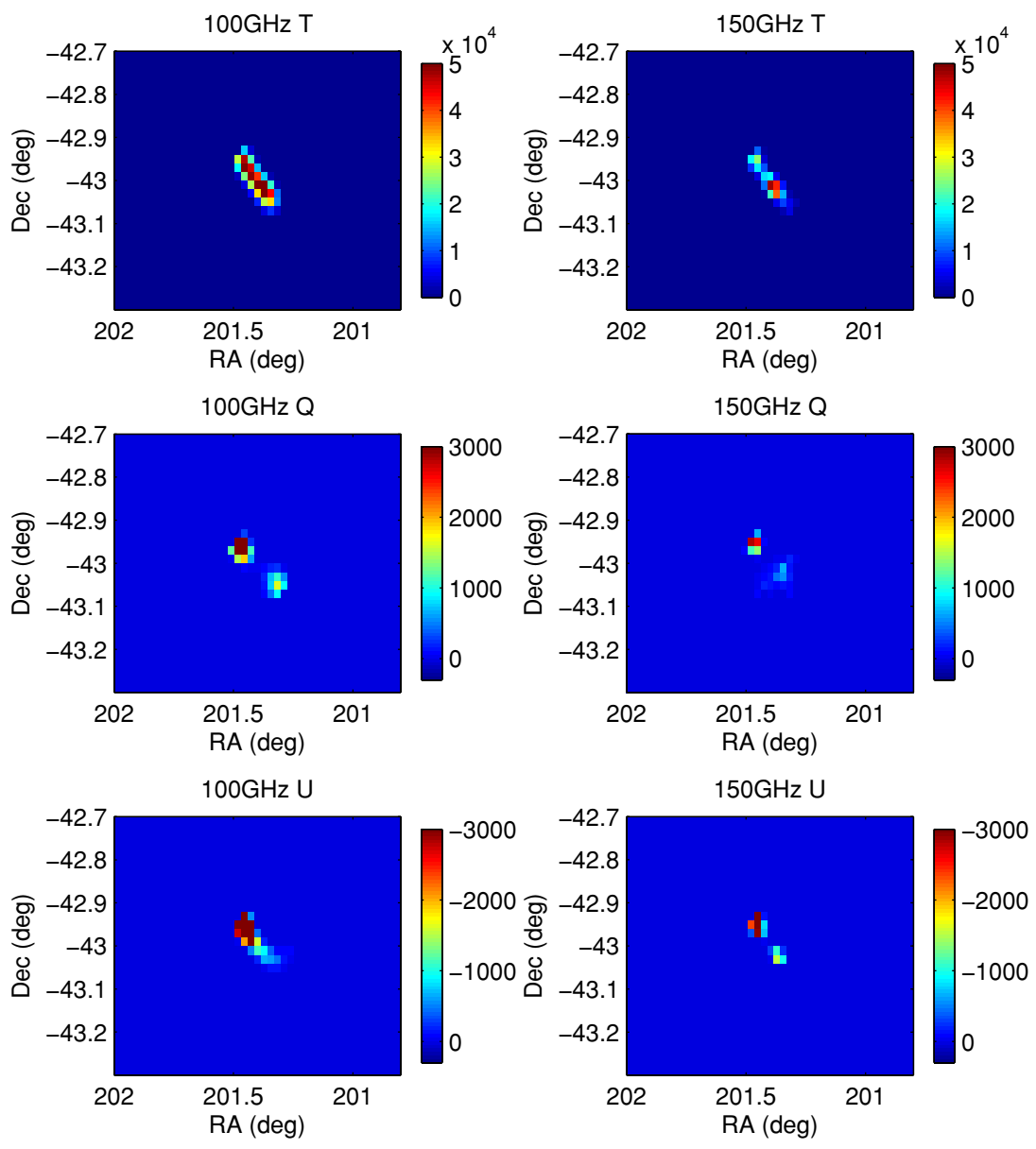

Figure 3. RLD maps of Centaurus A. The deconvolution kernel is a symmetric Gaussian with an FWHM matching the measured QUaD beam. Rows correspond to $T$ (top), $Q$ (middle), and $U$ (bottom) while columns are for $100 \mathrm{GHz}$ (left) and $150 \mathrm{GHz}$ (right). Temperature scale is $\mu \mathrm{K}_{\mathrm{CMB}}$; the scaling on the $U$ maps has been reversed to allow comparison with the $Q$ maps. Note that the deconvolved maps resolve the source into two distinct lobes with little or no polarized emission arising from the nuclear region between them, particularly at $150 \mathrm{GHz}$.

(A color version of this figure is available in the online journal.)

\subsection{Polynomial Filter Correction}

A second correction that needs to be applied to these data is due to the time series polynomial filter. The filter mask discussed in Section 3 is constructed as a compromise between maximizing the mask size to exclude as much source flux as possible from the polynomial filter fit and the need to minimize the mask so that individual scans are well constrained when the polynomial function is determined from the data. Although the mask effectively excludes the bright source flux during the polynomial filter fit, there always remains some small fraction of the source flux outside the mask for the scan length used in these measurements. This flux has the effect of biasing the filter determined from the data; this can be quantified using the $\mathrm{QUaD}$ simulator.

In order to produce realistic simulations of these observations, it is necessary to create an accurate source map at each polarization and frequency. Such a map needs to have high resolution so that the effect of the $\mathrm{QUaD}$ beams can be accurately simulated, and must have large enough signal-to-noise ratio on all components of the source that the input map is injecting negligible error into the simulation. In the case of the CMB, highly accurate simulations of the background sky based on known physical mechanisms at the epoch of recombination exist; unfortunately, the same is not true of Cen A. Such a map of the Cen A field at QUaD's frequencies can be produced either by scaling a map from a different frequency by the relevant spectral index of the source, or by using the QUaD maps themselves. The former method is difficult: maps of the Cen A region at radio frequencies tend to either have much higher resolution but are not sensitive to extended emission, or much lower resolution which would not allow us to resolve the source. We therefore have utilized the latter approach.

In order to increase the angular resolution of the Cen A maps, an algorithm that deconvolves the effective beam from the observed maps is necessary. We have chosen the RichardsonLucy deconvolution (RLD) algorithm here as it can efficiently deconvolve a known kernel from noisy data (Richardson 1972; Lucy 1974). The RLD algorithm employs Bayes' theorem to iteratively reconstruct the maximum likelihood background map given the beam kernel and the observed map with which it has been convolved. For the QUaD measurements of Cen A, the input maps are the observed 4 day co-added maps shown in Figures 1 and 2.

The effective QUaD beam for these observations has been calculated using noiseless simulations whose input is a delta function. These computed beams are well matched to a Gaussian to a level below $-15 \mathrm{~dB}$, so for computational ease we choose an RLD beam kernel to be a symmetric Gaussian with FWHM matching the $\mathrm{QUaD}$ beams at either frequency (H09). Applying the RLD algorithm to the 4 day co-added Cen A images yields the maps shown in Figure 3. The deconvolution is performed for each polarization state at both frequencies; in the case of 

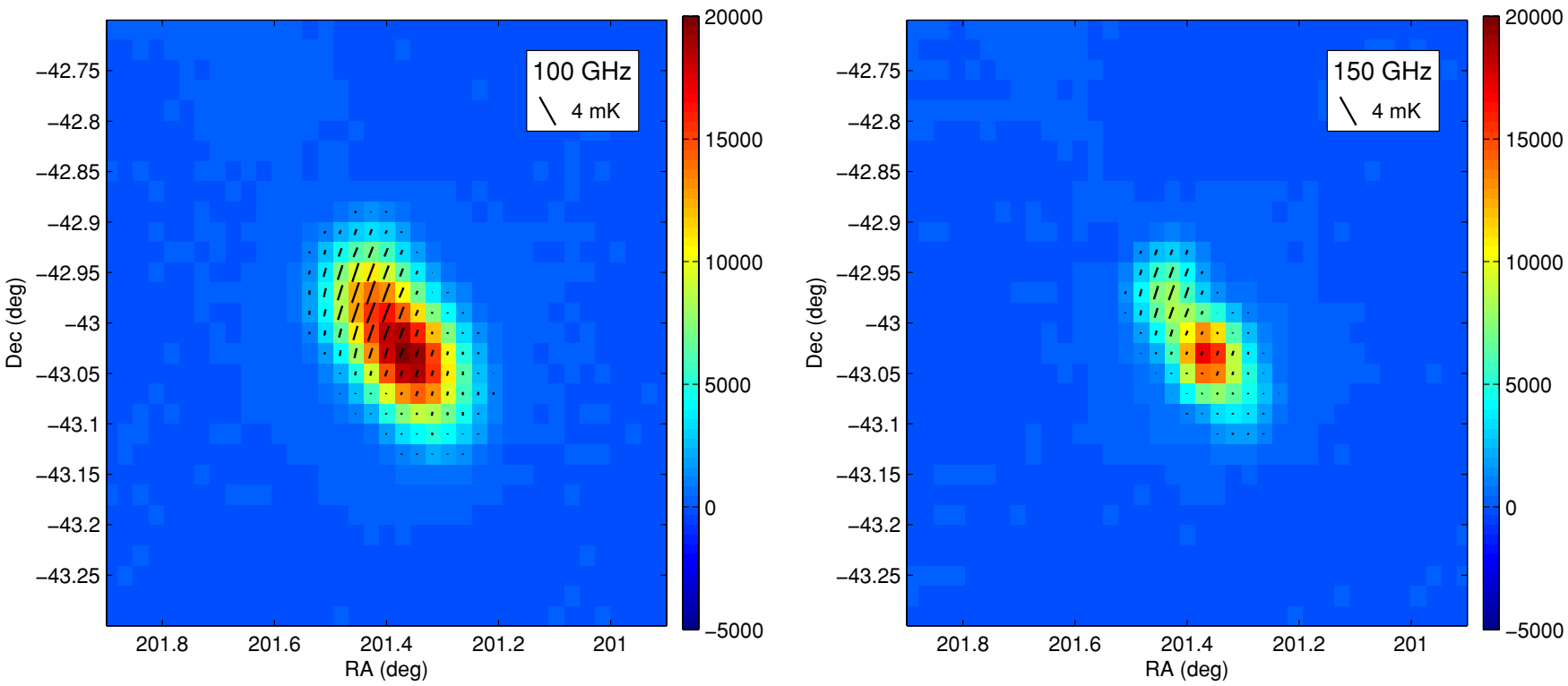

Figure 4. Final $T$ maps with fully processed polarization vectors overplotted. The left- and right-hand panels show the 100 and $150 \mathrm{GHz}$ maps, respectively. Polarization vectors are plotted for those map pixels with $T>1000 \mu \mathrm{K}$. The color scale is in $\mu \mathrm{K}$ and the length of a $4 \mathrm{mK}$ pure $+U$ vector is indicated.

$Q$ and $U$ the polarization, leakage corrected maps are used in the deconvolution so that polarization leakage need not be simulated.

The polynomial filter correction is determined by performing simulations similar to those discussed in the previous section. The RLD input maps are convolved with the ideal instrument response function at each frequency and TOD are constructed for each detector using the known telescope pointing. These TOD are then summed and differenced pair-wise, and a fifthorder polynomial is fit and subtracted from each pair's time streams. These filtered TOD are then binned in the usual way to produce fully corrected polarization maps; these constitute QUaD's final processed maps of Cen A.

\section{RESULTS}

Figure 4 shows the 4 day co-added maps after the systematic corrections have been applied. Using the RLD algorithm, these can be deconvolved with the nominal QUaD beam to remove the effect of the instrument transfer function to yield the maps similar to those shown in Figure 3; these maps allow simulations of this source in $I, Q$, and $U$ for any instrument with beam size similar to QUaD's or larger. ${ }^{15}$

A quantity which encapsulates a great deal about these polarization maps is the total $Q$ and $U$ in an aperture. Here we employ a circular aperture with $r=0.2$ centered on Cen A's position; this matches the source mask used during the time stream polynomial filtering. The total polarized temperatures for the final 4 day co-added maps are given by

$$
\mathcal{P}=\left\{\sum_{r} Q(\alpha, \delta), \sum_{r} U(\alpha, \delta)\right\} .
$$

The values of the aperture sums $\mathcal{P}$ measured from the QUaD maps are listed in Table 1 . The error estimate from the map, denoted $\sigma_{\text {map }}(\mathcal{P})$, is computed by calculating the rms of pixels in the same circular aperture offset from the source by $\Delta$ R.A. =

\footnotetext{
15 Both systematic corrected as-measured and RLD maps, including estimates of the noise in each map pixel, will be publicly available on the web at http://find.uchicago.edu/quad/quad_CenA/.
}

Table 1

Polarized Flux of Cen A from Corrected QUaD $Q$ and $U$ Maps

\begin{tabular}{lcc}
\hline \hline & $100 \mathrm{GHz}$ & $150 \mathrm{GHz}$ \\
\hline$\sum_{r} Q$ & $1.00 \mathrm{Jy}$ & $0.80 \mathrm{Jy}$ \\
$\sigma_{\text {map }}\left(\sum Q\right)$ & $0.06 \mathrm{Jy}$ & $0.05 \mathrm{Jy}$ \\
$\sigma_{\text {sim }}\left(\sum Q\right)$ & $0.06 \mathrm{Jy}$ & $0.06 \mathrm{Jy}$ \\
$\sum_{r} U$ & $-1.72 \mathrm{Jy}$ & $-1.40 \mathrm{Jy}$ \\
$\sigma_{\operatorname{map}}\left(\sum U\right)$ & $0.08 \mathrm{Jy}$ & $0.05 \mathrm{Jy}$ \\
$\sigma_{\text {sim }}\left(\sum U\right)$ & $0.06 \mathrm{Jy}$ & $0.07 \mathrm{Jy}$ \\
$\theta$ & -30.0 & -29.1 \\
$\sigma(\theta)$ & 0.9 & 1.2 \\
\hline
\end{tabular}

0.5 and dividing by the square root of the number of map pixels in the sum region. The error estimates $\sigma_{\text {map }}(\mathcal{P})$ are computed separately for $Q$ and $U$ at both frequencies.

An independent measurement of the error in $\mathcal{P}$ can be provided by the QUaD simulator, which includes an extremely accurate model of the noise in $\mathrm{QUaD}$ data. As in previous simulations, the RLD input map is convolved with the individual detector's beams and sampled with the telescope pointing. Based on each detector's statistical properties over a noise measurement block, noise is generated and injected into the scan TOD. P09 details the noise model construction in detail; it is useful to note here that the noise estimate for each detector is constructed from statistical blocks of that detector's actual time series 5 scans long. These time streams are then fifth-order polynomial filtered and binned into $I, Q, U$ maps as with the observed data. This simulation process is repeated $N$ times to obtain $N$ random realizations of the measurement. The scatter of $\mathcal{P}$ in these $N$ realizations yields the variation in the possible measurement outcomes due to the random noise in the data

Figure 5 shows the results of 256 such realizations for each day of observation. The standard deviations of these distributions are consistent with the aperture rms of the individual day maps, showing that the noise model is in good agreement with the variance in the data. Also plotted in this figure are the corrected per day $\mathcal{P}$ Cen A measurements; the hypothesis that these measurements are drawn from the same distribution as the simulated sample can be checked using Student's $t$-test. In the sample 

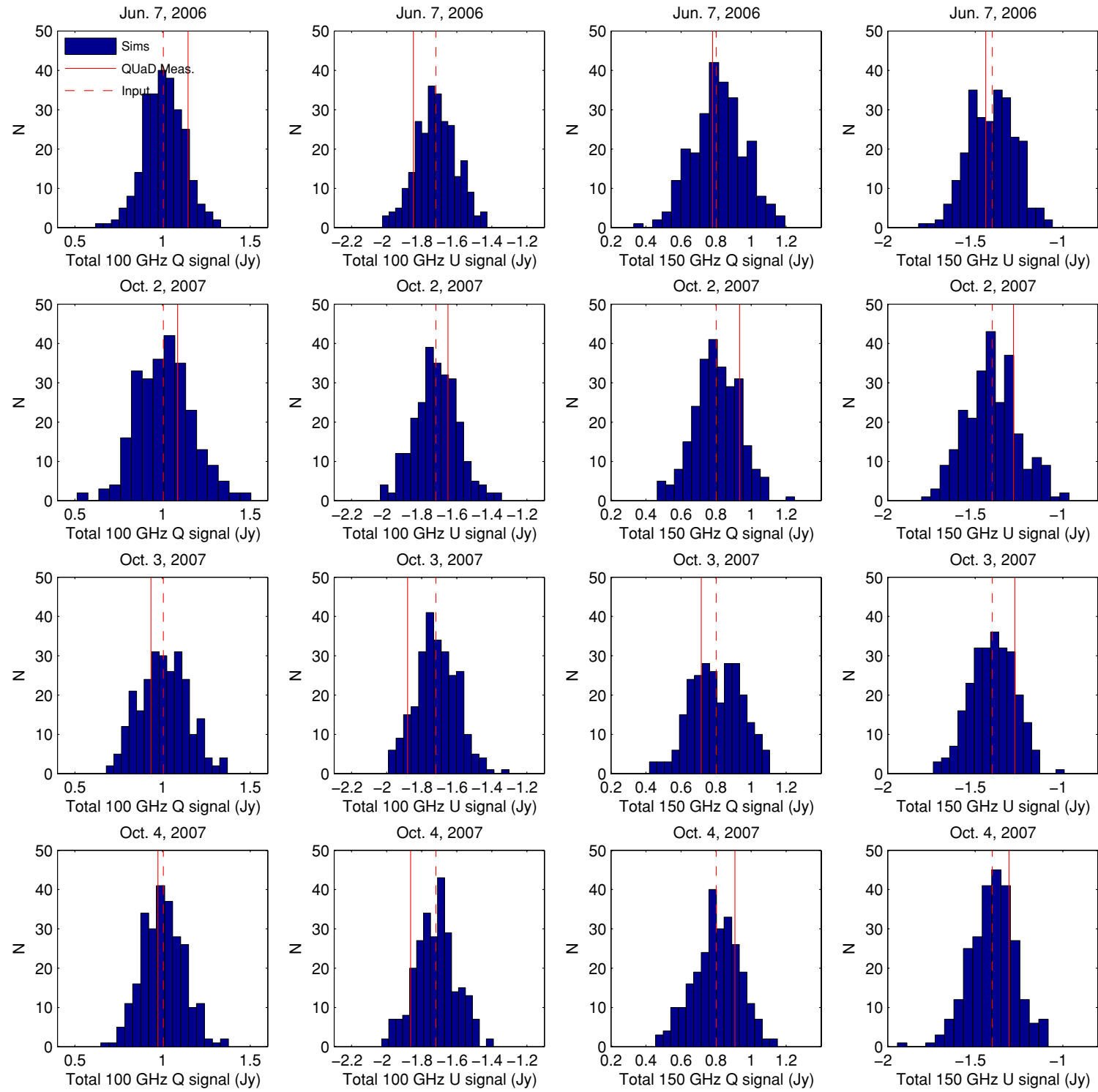

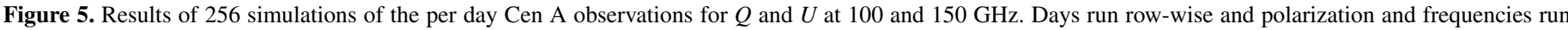

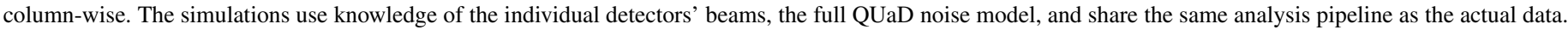

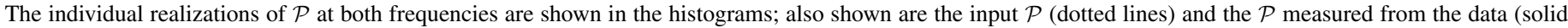

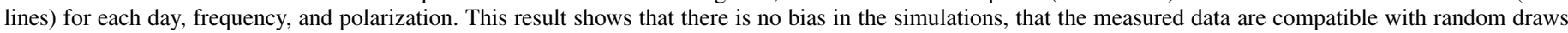
from such realizations, and that the noise in $\mathcal{P}$ can be estimated by the scatter in the simulation realizations.

(A color version of this figure is available in the online journal.)

of 16 different permutations of frequency, $Q, U$, and day, the null hypothesis is never rejected. This result is consistent with the null hypothesis for the overall set, and shows both that we have not detected a bias in the individual days' data and that the variance in the simulations is a realistic model for the variance in the real data. The random error estimate on $\mathcal{P}$ derived from the scatter of the simulation results is listed in Table 1 as $\sigma_{\text {sim }}(\mathcal{P})$.

The overall polarization angle of the source can be computed using

$$
\theta=\frac{1}{2} \arctan \left(\sum U / \sum Q\right)
$$

The values obtained in the $\mathrm{QUaD}$ measurement are listed in Table 1, including the random error $\sigma(\theta)$. The released data maps and Equation (4) can be applied per map pixel to determine the polarization angle variation over the source.

\section{SYSTEMATIC ISSUES}

There are a number of systematic effects that can add error to the measurement of $\mathcal{P}$ and $\theta$. These broadly group into either astrophysical effects or instrumental effects; these are discussed below.

\subsection{Background Structure}

The background structure in the Cen A field can potentially cause significant systematic error in this measurement. A number of measurements have mapped out Cen A's $8^{\circ} \times 4^{\circ}$ radio emitting outer lobes at various frequencies, most recently at high radio frequencies with WMAP (Wright et al. 2009). Israel et al. (2008) show detailed WMAP maps of Cen A at 23, 33, 41, 61, and $94 \mathrm{GHz}$; the outer lobe emission is clearly visible at the lower frequencies but is not significantly detected at $94 \mathrm{GHz}$. This result is evidence that at the QUaD frequencies 

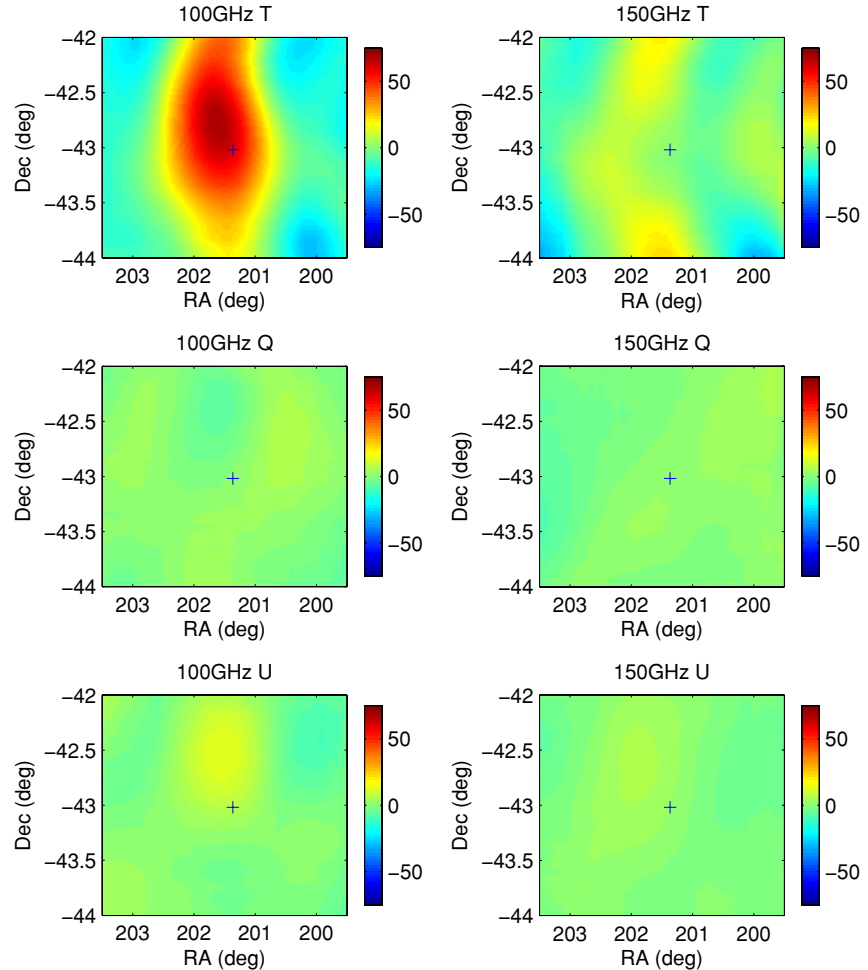

Figure 6. Background-filtered maps of the Cen A region. The left-hand column shows $100 \mathrm{GHz}$ maps for $T$ (top), $Q$ (middle), and $U$ (bottom); the right-hand column is the same for the $150 \mathrm{GHz}$ maps. Color scale is in $\mu \mathrm{K}$ and the cross in each map shows the nominal position of Cen A. The $100 \mathrm{GHz} T$ map is the only one exhibiting evidence of background structure; there is no evidence for background contamination in the polarization maps.

(A color version of this figure is available in the online journal.)

Cen A's outer lobe synchrotron emission is much below the brightness of the inner lobe region, but this result should be checked.

To perform this assessment, maps are first made in the standard way, except a first-order polynomial filter is used to remove the instrumental drifts from the time series of each detector; unlike the standard fifth-order polynomial used in the normal map making process, this filter preserves structures larger than $1^{\circ}$ on the sky. The central $r<0.2$ region is again masked during the polynomial fitting procedure to avoid bias. These data are binned into maps, and then the central polynomial mask region is replaced with the mean of the pixels in the annulus $0.2<r<0.3$ just beyond the mask region. These new maps are then convolved with a Gaussian kernel with an FWHM of $1^{\circ}$; this smooths out the small features whilst retaining the largest structures in the map. Figure 6 shows the resulting maps for $T, Q$, and $U$. Of these, only the $100 \mathrm{GHz} T$ map shows evidence for background structure on scales $\sim 1^{\circ}$. The total flux in the region where $T>20 \mu \mathrm{K}$ in this background-filtered $100 \mathrm{GHz}$ map is $10 \%$ of the total flux in the inner lobe region at $100 \mathrm{GHz}$. A number of different kernel sizes from 0.2 to $2^{\circ}$ have been applied in this procedure and the total flux in the background region does not change appreciably under different kernel widths. Table 2 lists the estimated uncertainty in $\mathcal{P}$ and $\theta$ caused by the background structure; for each polarization and frequency this quantity is calculated by summing the background signal in the region where $T_{100 \mathrm{GHz}}>20 \mu \mathrm{K}$ and multiplying the total by the ratio of the area of the $r<0.2$ region to the area of the summed region.

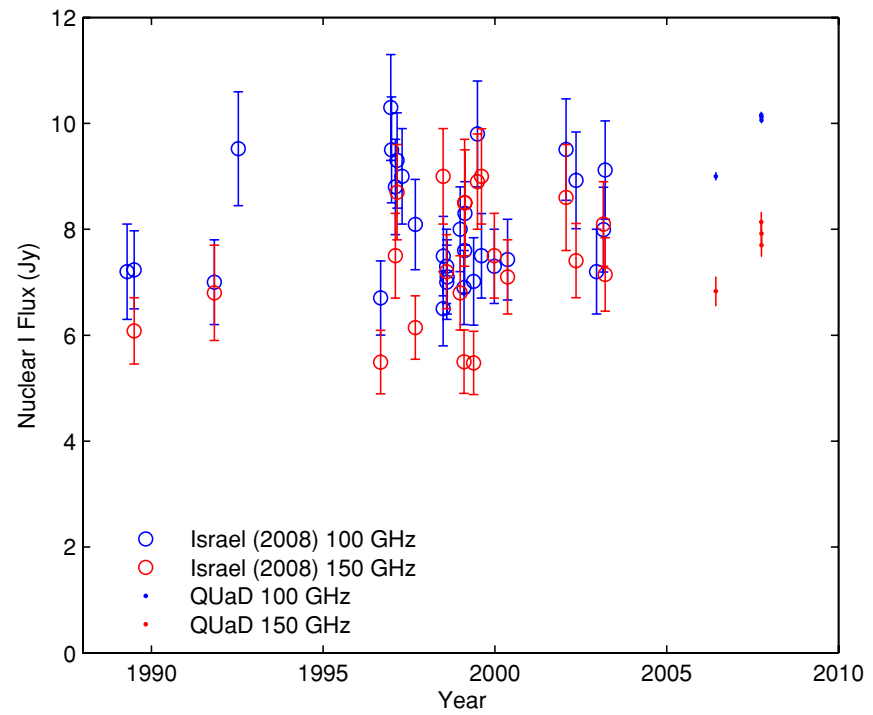

Figure 7. Variability in Centaurus A's nucleus at 100 and $150 \mathrm{GHz}$ for Stokes' $I$. The open circles show data presented in Israel et al. (2008) for the period 1988-2004 obtained using a heterodyne system on the SEST telescope, while the dots show the QUaD data presented here. These data are compatible with the well-known $\sim 30 \%$ peak to peak radio variation of this source at these wavelengths.

(A color version of this figure is available in the online journal.)

\subsection{Source Variability}

Another potential source of systematic error is temporal flux variability in Cen A itself. Although the inner lobe region is too large for its emission to change on human timescales, the nucleus of the source is known to be variable in $I$. Further, no measurements of this source's variability in polarization around $100 \mathrm{GHz}$ exist.

To measure the $I, Q$, and $U$ variability in Cen A, the nominal QUaD beam shape centered on the Cen A nucleus is fit to the final processed beam convolved maps at each frequency. As the size of Cen A's nucleus is much smaller than a QUaD beam, this fit yields the flux in the nuclear region, and subtracting it from the map yields the total flux in the inner lobe region for each frequency and polarization. The error in the resulting nuclear flux is calculated by measuring the rms deviations of the pixels in a number of circular apertures which have the same angular extent as a QUaD beam. The set of aperture rms statistics is then averaged to yield the noise in the measurement of the flux in a single aperture. Figure 7 shows the $I$ measured by QUaD in Cen A's nuclear region for each day of observation at 100 and $150 \mathrm{GHz}$. At both QUaD's bands, the I flux from Cen A's nucleus is $\sim 35 \%$ of the total flux from the inner lobe and nuclear regions; this implies that the temporal variability expected in the QUaD maps is $\lesssim 10 \%$ in $I$. Also plotted are the nuclear fluxes measured by Israel et al. (2008). These measurements were performed with a heterodyne system on SEST at several frequencies between 80 and $300 \mathrm{GHz}$ : in order to create data comparable to the QUaD set, we compute the QUaD bandpass-weighted average of the SEST points.

The Israel et al. (2008) points clearly exhibit the $\approx 30 \%$ peak to peak variation in $I$ well known from measurements at lower radio frequencies. The QUaD measurements also exhibit these fluctuations, although essentially only at two independent times as the 2007 measurements were performed on 3 consecutive days. These data do place a limit on the short term (day time 
Table 2

Cen A Measurement Uncertainty Budget

\begin{tabular}{|c|c|c|c|c|c|c|}
\hline Error on Parameter & $\sum Q_{100 \mathrm{GHz}}$ & $\sum U_{100 \mathrm{GHz}}$ & $\sum Q_{150 \mathrm{GHz}}$ & $\sum U_{150 \mathrm{GHz}}$ & $\theta_{100 \mathrm{GHz}}$ & $\theta_{150 \mathrm{GHz}}$ \\
\hline Beam pair offsets & $9 \mathrm{mJy}$ & $13 \mathrm{mJy}$ & $10 \mathrm{mJy}$ & $18 \mathrm{mJy}$ & 0.15 & 0.22 \\
\hline Differential beam shape & $4 \mathrm{mJy}$ & $5 \mathrm{mJy}$ & $6 \mathrm{mJy}$ & $6 \mathrm{mJy}$ & 0.06 & 0.11 \\
\hline Relative pair polarization angle & $9 \mathrm{mJy}$ & $6 \mathrm{mJy}$ & $5 \mathrm{mJy}$ & $4 \mathrm{mJy}$ & 0.12 & 0.09 \\
\hline Background structure & $1 \mathrm{mJy}$ & $0 \mathrm{mJy}$ & $4 \mathrm{mJy}$ & $32 \mathrm{mJy}$ & 0.01 & 0.29 \\
\hline Source variability & $14 \mathrm{mJy}$ & $31 \mathrm{mJy}$ & $56 \mathrm{mJy}$ & $68 \mathrm{mJy}$ & 0.28 & 1.05 \\
\hline Calibration angle & $35 \mathrm{mJy}$ & $35 \mathrm{mJy}$ & $28 \mathrm{mJy}$ & $28 \mathrm{mJy}$ & 0.50 & 0.50 \\
\hline Total systematic & $39 \mathrm{mJy}$ & $49 \mathrm{mJy}$ & $64 \mathrm{mJy}$ & $83 \mathrm{mJy}$ & 0.61 & $1: 23$ \\
\hline Random & $65 \mathrm{mJy}$ & $57 \mathrm{mJy}$ & $64 \mathrm{mJy}$ & $70 \mathrm{mJy}$ & 0.91 & 1.17 \\
\hline Total error & $76 \mathrm{mJy}$ & $75 \mathrm{mJy}$ & $91 \mathrm{mJy}$ & $109 \mathrm{mJy}$ & 1.09 & 1.69 \\
\hline
\end{tabular}
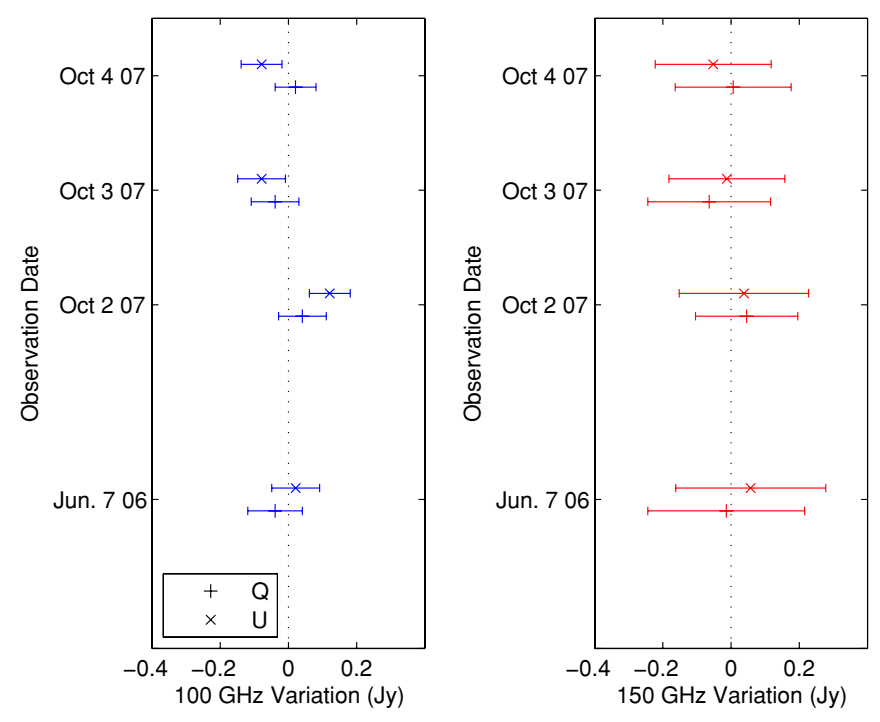

Figure 8. Time variability of Cen A in total $Q$ and $U$ at 100 and $150 \mathrm{GHz}$ (left and right, respectively). Symbol " + " shows $Q$ and symbol " $\times$ " shows $U$ for each of the four days of measurement; flux is given in Jy. The mean of each frequency and polarization group has been subtracted to isolate the variable component of the flux. As these points are consistent with no time variation, half of their peak to peak scatter is used as the error associated with variability in the measurement of Cen A's polarization properties.

(A color version of this figure is available in the online journal.)

scale) $I$ variability of the Cen A nucleus of $1 \%$ at $100 \mathrm{GHz}$ and $6 \%$ at $150 \mathrm{GHz}$. The $I$ flux of only the inner lobes in the standard $r<0.2$ aperture is measured to be $14.2 \pm 0.3 \mathrm{Jy}$ at $100 \mathrm{GHz}$ and $16.6 \pm 1.1 \mathrm{Jy}$ at $150 \mathrm{GHz}$.

Cen A's nuclear flux in $Q$ and $U$ is computed in the same way as for $I$; Figure 8 shows the resulting $Q$ and $U$ for the 4 days of observation. Comparing to the model that the flux of these 16 measurements is constant, the obtained reduced $\chi^{2}=0.64$; based on the $\mathrm{QUaD}$ data, there is no evidence of polarized temporal variability in Cen A. An upper limit on the possible variation can be obtained by computing the mean absolute deviation of these individual day data from their mode; the limits are listed in Table 2 . These limits are significantly smaller than the estimated 30\% variation expected from the $I$ variability. Inspection of the RLD maps in Figure 3 shows that very little of the polarized emission is originating from the nuclear region itself. This is evidence that this source's polarization stability arises because the polarized millimeterwave flux is predominantly being emitted by the inner lobe region rather than the nucleus.

\subsection{Instrumental Parameters}

There are a number of instrumental parameters used in the QUaD analysis pipeline that can cause systematic errors; a review of these is given in P09. Following that work, for these Cen A data the possible systematic errors caused by misestimates of these parameters are investigated using the QUaD simulator.

The absolute calibration uncertainty from detector output to $T_{\mathrm{CMB}}$ is estimated to be $3.4 \%$ (Brown et al. 2009). As this uncertainty is completely correlated between $I, Q$, and $U$ it does not affect polarization angle or fraction, only absolute polarization temperature. For this reason it is omitted from Table 2, though formally it should be included in a complete accounting of the uncertainty in Cen A's $Q$ and $U$.

As discussed in Section 4.1, the detector pair beam offsets cause leakage of $I$ to polarization. Although we have accounted for this effect, the measurement of the offsets is still subject to error. To measure the effect of a random misestimation of the detector offsets, we have performed simulations where the measured detector offsets are randomized by the rms centroid uncertainty of $0{ }^{\prime} 15$. Though this leads to no change in the mean value of $\mathcal{P}$, it increases the noise in the measurement by a few tenths of $\mathrm{mK}$. We adopt the simulated values as a conservative estimate of the error due to misestimation of the detector offsets in Table 2.

Although the polarized beam shape $\mathcal{B}$ has been measured to be symmetric between bolometers sharing a feed, the error of this measurement is approximately $2.5 \%$ of the beam width. The uncertainty in the individual detector beam widths could cause a systematic error in this analysis. As there is no evidence for a systematic shift in the beam widths, to quantify this error we have performed simulations where the pair beam widths are scattered about their known values using a Gaussian randomization with $1 \sigma=2.5 \%$. In addition, the relative detector pair polarization angle uncertainty is also a potential source of polarization error. Simulations are performed assuming a $1 \sigma=1^{\circ}$ Gaussian scatter on the measured detector pair relative angles, which is the estimated per pair uncertainty on $\psi$ (see H09). The results of both of these simulation sets are listed in Table 2.

As discussed in $\mathrm{P} 09$, we estimate the polarization leakage of our detectors to be $\epsilon=0.08 \pm 0.015$. Random errors in $\epsilon$ will average down, while a systematic change merely shifts the absolute calibration by a factor of $\sim 2 \sigma_{\epsilon}$. As the uncertainty on $\epsilon$ is $<0.02$ this is sub-dominant to the uncertainty on the overall absolute calibration. Sidelobe pickup of polarized emission from the ground may also be a concern (Brown et al. 2009). However, both the use of the fifth-order polynomial filter and the small 
angular extent of the source suppress the magnitude of this effect to the $<10 \mu \mathrm{K}$ level, so it is not a significant source of systematic error in this measurement.

Table 2 summarizes the error budget for this measurement including all important systematic errors. The total systematic and total errors have been calculated using the square root of the quadrature sum of each of the individual errors.

\section{CONCLUSION}

We have presented measurements of the $4^{\circ} \times 2^{\circ}$ region centered on the radio source Centaurus A with QUaD, a millimeterwave polarimeter whose absolute polarization angle is known to an accuracy of \pm 0.5 . Systematic errors from astronomical sources such as the Cen A field's background structure and temporal variability from the source's nuclear region have been estimated. Simulations have been performed to assess the effect of misestimation of the instrumental parameters on the final measurement. After correcting for known instrumental effects, we find that the total $(Q, U)$ of the inner lobe region is $(1.00 \pm 0.08,-1.72 \pm 0.08) \mathrm{Jy}$ at $100 \mathrm{GHz}$ and $(0.80 \pm 0.09,-1.40 \pm 0.11) \mathrm{Jy}$ at $150 \mathrm{GHz}$, leading to polarization angles of $-30.0 \pm 1.1$ and $-29.1 \pm 1.7$.

Future millimeter polarimeters, including CMB experiments, will require a well-characterized astronomical source against which to compare their instruments' laboratory determined polarization properties. Cen A represents one of the best candidates for a stable, compact, highly polarized source, and the measurements presented here will allow calibration of such instruments to an uncertainty of $\sim 1^{\circ}$.
QUaD is funded by the National Science Foundation in the USA, through grants ANT-0338138, ANT-0338335, and ANT0338238, by the Science and Technology Facilities Council (STFC) in the UK and by the Science Foundation Ireland. The BOOMERanG collaboration kindly allowed the use of their CMB maps for our calibration purposes. M.Z. acknowledges support from a NASA Postdoctoral Fellowship. P.G.C. acknowledges funding from the Portuguese FCT. S.E.C. acknowledges support from a Stanford Terman Fellowship. J.R.H. acknowledges the support of an NSF Graduate Research Fellowship, a Stanford Graduate Fellowship, and a NASA Postdoctoral Fellowship. Y.M. acknowledges support from a SUPA Prize studentship. C.P. acknowledges partial support from the Kavli Institute for Cosmological Physics through the grant NSF PHY0114422. E.Y.W. acknowledges receipt of an NDSEG fellowship.

\section{REFERENCES}

Barkats, D., et al. 2005, ApJS, 159, 1

Brown, M. L., et al. 2009, ApJ, 705, 978

Hamaker, J. P., \& Bregman, J. D. 1996, A\&AS, 117, 161

Hinderks, J. R., et al. 2009, ApJ, 692, 1221 (H09)

Hivon, E., Górski, K. M., Netterfield, C. B., Crill, B. P., Prunet, S., \& Hansen, F. 2002, ApJ, 567, 2

Israel, F. P. 1998, A\&AR, 8, 237

Israel, F. P., Raban, D., Booth, R. S., \& Rantakyrö, F. T. 2008, A\&A, 483, 741

Leitch, E. M., et al. 2002, Nature, 420, 763

Lucy, L. B. 1974, AJ, 79, 745

Pryke, C., et al. 2009, ApJ, 692, 1247 (P09)

Richardson, W. H. 1972, J. Opt. Soc. Am., 62, 55

Wright, E. L., et al. 2009, ApJS, 180, 283 